\title{
BRIEF
}

\section{Description and Evaluation of a Pilot Advanced Pharmacy Practice Experience in Addiction Medicine}

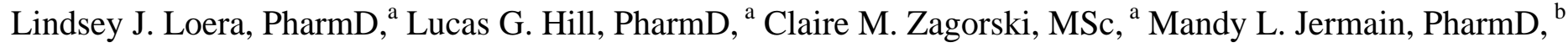 \\ Carlos F. Tirado, MD, MPH ${ }^{\mathrm{c}}$ \\ ${ }^{a}$ The University of Texas at Austin, College of Pharmacy, Austin, Texas \\ ${ }^{\mathrm{b}}$ Pharmacyclics, An AbbVie Company, Silicon Valley, California \\ ${ }^{c}$ CARMAhealth PLLC, Austin, Texas
}

Corresponding Author: Lindsey J. Loera, The University of Texas at Austin, College of Pharmacy, 2409 University Ave, Austin, TX 78712. Tel: 512-496-2728. Email: Lindsey.Loera@austin.utexas.edu

Submitted October 20, 2021; accepted February 1, 2022; ePublished February 2022

Objective. To assess the impact of a pilot advanced pharmacy practice experience (APPE) on fourth year (P4) Doctor of Pharmacy (PharmD) students' knowledge and confidence related to substance use disorders (SUD), harm reduction, and co-occurring psychiatric conditions.

Methods. Beginning in 2020, a 62-item assessment was developed and administered to P4 students at the beginning and end of the six-week APPE. The assessment tested knowledge in 10 content areas related to SUD, harm reduction, and cooccurring disorders. Students also ranked their confidence providing care related to each content area. The postassessment included a free text item to provide feedback on the APPE experience. Descriptive statistics and paired t-tests were used to analyze the data.

Results. Complete pre- and post-assessments were obtained from all participating students $(\mathrm{n}=7)$. The mean cumulative knowledge score increased from $55.2 \%$ to $81.5 \%$ and the mean cumulative confidence score improved from $34.2 \%$ to $81.8 \%$. Free text responses garnered positive feedback from students stating the APPE allowed them to immerse themselves in all stages of the recovery process, increase confidence in presentation skills with patients, and solidified their passion for addiction medicine.

Conclusion. A novel APPE in addiction medicine addressed a current gap in pharmacy education, earned positive evaluations from student pharmacists, increased student knowledge and confidence related to SUD, harm reduction, and co-occurring disorders, and supported the development of new interprofessional collaborations. United States colleges of pharmacy that do not yet offer APPEs in this clinical domain should consider this model.

Keywords: pharmacists, addiction medicine, pharmacy education

\section{INTRODUCTION}

The United States (U.S.) faces a substantial burden of societal harms related to substance use disorder (SUD). Illegal drug use has continued to rise since 2015 with 57.2 million people reporting illegal drug use in $2019 .{ }^{1}$ Drug overdoses and drug overdose mortality increased dramatically from 2019 to 2020 , largely due to synthetic opioids. ${ }^{2}$ As the most accessible healthcare provider, pharmacists are uniquely positioned to address this public health issue but must be adequately trained to do so effectively.

In 2020, the American Association of Colleges of Pharmacy's (AACP) Special Committee on Substance Use and Pharmacy Education stated, "Experiential opportunities to apply foundational SUD knowledge in clinical environments must be developed and supported." ${ }^{3}$ A 2015 survey of 75 AACP member schools found that 79\% provided at least two hours of required didactic content related to SUD and 30\% provided at least four hours. ${ }^{4}$ However, advanced pharmacy practice experiences (APPEs) focused on SUD treatment were available in only 23\% of programs and even then were offered to a mean of only 2.6 students each year. ${ }^{5}$ The 2019 AACP environmental scan of opioid-related activities identified many new educational and service opportunities for students related to opioid overdose education and naloxone distribution. However, very few submissions described SUD-related APPEs. ${ }^{6}$

The University of Texas at Austin College of Pharmacy offers a required curriculum that includes 20 hours of didactic instruction related to SUD treatment and harm reduction. However, prior to 2019, fourth year (P4) doctor of pharmacy (PharmD) students could gain clinical exposure to SUD only as a secondary aspect of psychiatry-focused APPEs. In the summer of 2019, a new elective APPE in addiction medicine was piloted with two P4 PharmD students. In 
the summer of 2020, the offering was expanded to three students in a single six-week rotation block. In 2021, it further expanded into two six-week blocks with two students in each block. This paper describes the learning experiences offered in the 2020 and 2021 iterations and the associated learning outcomes.

\section{APPE Description}

A total of seven students selected through application and interview by the two pharmacist instructors participated in this elective APPE from 2020-2021. The APPE spanned six-weeks and totaled 250-260 hours of training for each student with a combination of experiences in patient care and research. APPE instructors included (1) a clinical pharmacist and faculty member with expertise in addiction medicine practice and research, (2) a physician with board certification in general psychiatry, addiction psychiatry, and addiction medicine who supported clinical integration, and (3) a post-PharmD fellow who participated in the APPE as a P4 in 2019 and contributed to program improvement and logistical coordination. The goal of the APPE was to increase students' knowledge and confidence related to SUD and psychiatric conditions while exposing them to a variety of interprofessional SUD care environments.

Practice sites in 2020 included (1) an outpatient medical home for SUD and co-occurring disorders, (2) an addiction treatment center offering services ranging from medically-supervised withdrawal to intensive outpatient programming, (3) a 90-day residential treatment center for parents seeking recovery, and (4) a county-funded opioid treatment program specializing in treatment with methadone. Additional practice settings added in 2021 included (1) a mobile outreach van offering weekly harm reduction services and (2) county emergency medical service program providing linkage to care for SUD and mental health. None of the practice sites had ever previously employed or otherwise engaged a pharmacist in the provision of direct patient care. The students were directly supervised by on-site clinicians including physicians, advanced practice nurses, and paramedics and engagement included supporting medication reconciliation, medication counseling, and patient interviewing. Students also led 45-minute interactive medication education presentations with clients every one to two weeks on the topics outlined in Table 3, as well as sexually transmitted infections, human immunodeficiency virus (HIV), and hepatitis C virus (HCV). Upon conclusion of the medication education presentation, 10 minutes were allotted for question and answer. The P4 students impacted 59 and 119 patients through these groups in 2020 and 2021 respectively.

Students were allotted time each Monday to work on assigned research projects before rotating through the practice sites Tuesday through Friday. Research projects in 2020 went on to presentation at a national conference and included (1) two statewide telephone audits of community pharmacies assessing availability of buprenorphine/naloxone films and naloxone nasal spray and (2) a survey of patients' satisfaction and experience with long-acting formulations of buprenorphine and naltrexone. ${ }^{7-9}$ Projects in 2021 included (1) a survey of persons in active drug use assessing current heroin mixing practices and (2) evaluation of changes in clinical characteristics of patients at the outpatient medical home over time.

Weekly learning assignments included (1) presentation and review of an assigned SUD-related journal article, (2) a summary of a patient interaction seen at the practice sites, (3) several informal presentations on minor topics, and (4) two topic discussions on a disorder/condition. All presentations were delivered via online video conference technology to the primary instructors and members of their research program. At the conclusion of each presentation, attendees were encouraged to ask questions and submit anonymous feedback.

\section{OBJECTIVE}

The goal of this analysis was to assess the impact of a pilot APPE on P4 PharmD students' knowledge and confidence related to SUD, harm reduction, and co-occurring psychiatric conditions.

\section{METHODS}

Beginning in 2020, a 62-item assessment was developed by the pharmacist APPE instructors to assess students' learning before and after the APPE. The assessment had 51 items to assess knowledge (47 short answer, 4 multiple choice) and 11 Likert-scale items to assess confidence. Knowledge was tested in 10 content areas which are described in Table 3. Short answer questions assessed knowledge regarding guideline recommendations, medication names, mechanism of action, dosing, and clinical assessment tools. Within each content area students ranked their confidence providing care to persons with that respective condition. Primary outcomes included assessing the change in students' knowledge and confidence from pre- to post-APPE. Secondary outcomes included the student-reported experience and feedback on the APPE, as well as intentions for post-graduate training. Descriptive statistics were used to analyze demographic variables and a paired t-test was used to evaluate changes in knowledge and confidence. The University of Texas at Austin Institutional Review Board determined this was not human subjects research. 


\section{RESULTS}

Complete pre- and post-assessments were obtained from all participating students $(n=7)$. Students were predominantly female $(85.7 \%)$ and White $(85.7 \%)$. The mean age of the students was 27 years (range 25-33) and all seven anticipated pursuing specialized residency training in psychiatric pharmacy (Table 1). The mean cumulative knowledge score increased from $55.2 \%$ to $81.5 \%$ ( $p<.001)$ and the mean cumulative confidence score improved from $34.2 \%$ to $81.8 \%$ $(p<.001)$ (Table 2). Free text responses garnered positive feedback from students stating the APPE allowed them to immerse themselves in all stages of the recovery process, increase confidence in presentation skills, and solidify their passion for SUD. When asked about the overall APPE experience, anecdotal responses included:

"My favorite part of the APPE was working with the mobile outreach van because meeting patients where they're at and being able to navigate local resources is a key aspect of the impact pharmacists can have in their communities."

"During medication education groups I was able to grow my confidence with presentations skills and my ability to discuss stigma or tough questions with clients. The insight from this APPE will help me tremendously in the future to better serve patients who are in recovery."

"I interacted with patients on the entire spectrum of SUD, from persons who actively use to those in long-term recovery. What I will carry with me into my future practice is the importance of creating connections with patients, treating them with respect, and refraining from judgement."

\section{DISCUSSION}

This is the first report, to our knowledge, to detail an APPE in addiction medicine. Participation in this pilot APPE was associated with increases in clinical knowledge and self-reported confidence providing care to patients with SUD and common comorbidities. In addition to serving as a positive signal regarding the value of the learning experiences in the APPE, these results demonstrate that an APPE in this emerging area of pharmacy practice can be successfully implemented without an on-site pharmacist preceptor. This may represent a more feasible model for many U.S. colleges of pharmacy that do not yet have clinical faculty in this domain. Providing substantial opportunities for experiential learning related to substance use and addiction in the P4 year should be a high priority for academic pharmacy. With adequate training, pharmacists can play a key role in SUD treatment and harm reduction. ${ }^{10}$ However, in the absence of such training, pharmacists may continue to represent a barrier to accessing evidence-based interventions rather than a facilitator. ${ }^{11,12}$

In 2021, the APPE was enhanced by the addition of clinical sites involving community paramedics and harm reductionists. Emergency medical service programs for persons who use drugs provide outreach and increase access to evidence-based care. ${ }^{13}$ Inclusion of such a program has the potential to increase student pharmacists' collaborative skills and increase their awareness of community resources. Syringe services programs (SSPs) which offer a range of harm reduction supplies and interventions represent another valuable addition to pharmacy education. SSPs are associated with reduced rates of HCV and HIV and can link persons to referral for SUD-treatment. ${ }^{14}$ Pharmacists must be knowledgeable and confident in making effective harm reduction recommendations to better serve patients.

Due to the success of the 2019 and 2020 iterations, the physician instructor and practice sites agreed to begin hosting four P4 students each year. Student exposure to non-pharmacologic therapies increased substantially from participation in only group processing and music therapy, to also include three types of behavioral therapy: contingency management, mindfulness-based relapse prevention, and the Matrix Model. This pilot APPE and the modifications detailed has enhanced the training of P4 students and supported the development of collaborative relationships with the physician instructor and practice sites. This has resulted in interprofessional scholarship, planning of larger scale research projects, and the development of a two-year post-PharmD fellowship with a clinical component. In future iterations, the APPE instructors intend for responsibilities of the post-PharmD fellow to include on-site preceptorship to the P4 students.

Evaluation of this APPE has several limitations. Of particular note is the small, demographically homogeneous participant sample from a single institution. Selection bias is also a factor given the high level of motivation to pursue a career in psychiatric pharmacy among the participating students. Whether these results would be maintained with a larger, more diverse sample is unknown. Additionally, while other U.S. colleges of pharmacy reported an average of 2.7 hours of required didactic instruction on SUD in 2015, The University of Texas at Austin College of Pharmacy curriculum provided 20 hours for 2021 PharmD graduates. ${ }^{4}$ It may be reasonable to assume the impact of an APPE in addiction medicine would be even more profound for students with less prior exposure.

\section{CONCLUSION}

This pilot APPE in addiction medicine increased student knowledge and confidence related to SUD, harm reduction, and psychiatric conditions and exposed students to a variety of interprofessional environments. It also earned positive student evaluations and supported the development of new interprofessional collaborations. U.S. colleges of 
pharmacy that do not yet offer APPEs in this clinical domain should consider this model to create optional SUD training opportunities for students.

\section{ACKNOWLEDGEMENTS}

This study was supported by grant 1H79TI081729-01 from the Substance Abuse and Mental Health Services Administration (SAMHSA). The funder had no role in the design and conduct of the study; collection, management, analysis, and interpretation of the data; preparation, review, or approval of the manuscript; and decision to submit the manuscript for publication. The content of this study does not represent the official view of SAMHSA or the Texas Health and Human Services Commission.

Dr. Tirado reports serving as a consultant for Alkermes, Inc and US World Meds, LLC. Dr. Hill reports serving on the Hikma Specialty Inc. Community Advisory Board in December 2020. No other authors have any potential conflicts of interest or funding to report.

\section{REFERENCES}

1. U.S. Department of Health and Human Services (HHS) - Substance Abuse \& Mental Health Services Administration. 2019 Survey on Drug Use and Health https://www.samhsa.gov/data/report/2019-nsduh-detailed-tables. Accessed June $14,2021$.

2. Centers for Disease Control and Prevention. Increase in Fatal Drug Overdoses Across the United States Driven by Synthetic Opioids Before and During the COVID-19 Pandemic. Updated December 17, 2020. Accessed June 14, 2021. https://emergency.cdc.gov/han/2020/han00438.asp.

3. Tran T, Ball J, Bratberg JP, et al. Report of the 2020 Special Committee on Substance Use and Pharmacy Education. Am J Pharm Educ. 2020;84(11):8421. doi:10.5688/ajpe8421.

4. Thomas K, Muzyk AJ. Surveys of substance use disorders education in US pharmacy programs. Ment Health Clin. 2018;8(1):14-7. doi:10.9740/mhc.2018.01.014.

5. Dopheide JA, Bostwick JR, Goldstone LW, et al. Curriculum in Psychiatry and Neurology for Pharmacy Programs. Am J Pharm Educ. Sep 2017;81(7):5925. doi:10.5688/ajpe8175925.

6. Bradley-Baker LR, Cipriani M, Farrell DP, et al. Environmental scan of opioid-related activities in academic pharmacy. Poster presented at: Rx Drug Abuse \& Heroin Summit; April 13-16, 2020; Nashville, TN.

7. Charles AN, Burgess MM, Vogt CM, et al. Maintaining momentum on the road to recovery: availability of buprenorphine/naloxone films and naloxone nasal spray in community pharmacies in Maine. Poster presented at: ASHP Midyear Clinical Meeting; December 6-10, 2020; Virtual.

8. Burgess MM, Charles AN, Vogt CM, et al. Maintaining momentum on the road to recovery: availability of buprenorphine/naloxone films and naloxone nasal spray in community pharmacies in Massachusetts. Poster presented at: ASHP Midyear Clinical Meeting; December 6-10, 2020; Virtual.

9. Vogt CM, Burgess MM, Charles AN, et al. Analysis of patient-reported satisfaction with long-acting injectables for opioid and alcohol use disorder in a central Texas outpatient clinic. Ment Health Clin. 11(2):79-80. doi:10.9740/mhc.2021.03.075.

10. Coon SA, Hill LG, Hutchison RW, et al. Mobilizing pharmacists to address the opioid crisis: a joint opinion of the ambulatory care and adult medicine practice and research networks of the American College of Clinical Pharmacy. $J$ Am Coll Clin Pharm. 2020;3:1493-1513.

11. Hill LG, Loera LJ, Evoy KE, et al. Availability of buprenorphine/naloxone films and naloxone nasal spray in community pharmacies in Texas, USA. Addiction. 2021;116(6):1505-1511. doi:10.1111/ADD.15314.

12. Evoy KE, Hill LG, Groff L, et al. Naloxone accessibility without a prescriber encounter under standing orders at community pharmacy chains in Texas. JAMA. 2018; 320:1934-1937

13. Langabeer JR, Persse D, Yatsco A, et al. A framework for EMS outreach for drug overdose survivors: a case report of the Houston emergency opioid engagement system. Prehosp Emerg Care. 2021; 25:3,441-448. doi:10.1080/10903127.2020.1755755.

14. Centers for Disease Control and Prevention. Syringe services programs fact sheet. Updated May 23, 2019. Accessed April 12, 2021. https://www.cdc.gov/ssp/syringe-services-programs-factsheet.html. 
Table 1. Characteristics of Participating Students

\begin{tabular}{lc}
\hline Characteristic & $\mathbf{N}(\boldsymbol{\%})$ \\
\hline Sex & $6(85.7)$ \\
Female & $1(14.3)$ \\
Male & $6(85.7)$ \\
Race/Ethnicity & $1(14.3)$ \\
$\quad$ White/Caucasian Only & 27 \\
Hispanic/Latino Only & $25-33$ \\
Age (median in years) & $7(100)$ \\
Age (range in years) & $7(100)$ \\
Bachelors Degree & \\
Anticipated Post-Graduate Training & \\
$\quad$ PGY1 + PGY2 Residency &
\end{tabular}

Table 2. Changes in Knowledge and Confidence Among Participating Students

\begin{tabular}{|c|c|c|c|}
\hline Condition & Pre-APPE Mean (\%) & Post-APPE Mean (\%) & $\%$ Increase \\
\hline Knowledge & 55.2 & 81.5 & $26.3^{\mathrm{a}}$ \\
\hline \multicolumn{4}{|l|}{2020} \\
\hline Student 1 & 35.3 & 76.5 & 41.2 \\
\hline Student 2 & 52.9 & 84.3 & 31.4 \\
\hline Student 3 & 43.1 & 76.5 & 33.4 \\
\hline \multicolumn{4}{|l|}{2021} \\
\hline Student 4 & 66.7 & 92.1 & 25.4 \\
\hline Student 5 & 58.8 & 82.4 & 23.6 \\
\hline Student 6 & 70.6 & 90.2 & 19.6 \\
\hline Student 7 & 58.8 & 68.6 & 9.8 \\
\hline Confidence & 34.2 & 81.8 & $47.6^{\mathrm{a}}$ \\
\hline \multicolumn{4}{|l|}{2020} \\
\hline Student 1 & 12.1 & 57.6 & 45.5 \\
\hline Student 2 & 36.4 & 78.8 & 42.4 \\
\hline Student 3 & 36.4 & 84.9 & 48.5 \\
\hline \multicolumn{4}{|l|}{2021} \\
\hline Student 4 & 42.4 & 84.8 & 42.4 \\
\hline Student 5 & 24.2 & 93.9 & 69.7 \\
\hline Student 6 & 45.5 & 81.8 & 36.3 \\
\hline Student 7 & 42.4 & 90.9 & 48.5 \\
\hline
\end{tabular}


Table 3. Breakdown of 62-Item Pre- and Post- APPE Assessment

Content Area
Neurobiology/Psychotherapies

\section{Item Breakdown $(\mathbf{N})^{\text {a }}$}

Short Answer (2)

Confidence Ranking (2)

Multiple Choice (4)

Alcohol Use Disorder

Opioid Use Disorder

Stimulant Use Disorder

Tobacco Use Disorder

Harm Reduction

MDD/GAD

Bipolar Disorder

Schizophrenia

Sleep Disorders
Short Answer (2)

Confidence Ranking (1)

Short Answer (4)

Confidence Ranking (1)

Short Answer (3)

Confidence Ranking (1)

Short Answer (3)

Confidence Ranking (1)

Short Answer (5)

Confidence Ranking (1)

Short Answer (13)

Confidence Ranking (1)

Short Answer (3)

Confidence Ranking (1)

Short Answer (5)

Confidence Ranking (1)

Short Answer (7)

Confidence Ranking (1)

Abbreviations: MDD=Major Depressive Disorder. GAD=Generalized Anxiety Disorder.

${ }^{\mathrm{a}}$ The respondents were asked to rate self-reported confidence of providing care in each content area on a Likert-type scale of 0 (very uncomfortable) to 3 (very confident). 